\title{
Sudden Cardiac and Noncardiac Death in Sports: Epidemiology, Causes, Pathogenesis, and Prevention
}

\author{
Giuseppe Lippi, MD ${ }^{1}$ Emmanuel J. Favaloro, PhD, FFSc (RCPA) ${ }^{2, *}$ Fabian Sanchis-Gomar, MD ${ }^{3,4, *}$ \\ ${ }^{1}$ Section of Clinical Biochemistry, Department of Neurological, \\ Biomedical and Movement Sciences, University of Verona, Verona, \\ Italy \\ 2 Department of Clinical and Laboratory Haematology, Sydney \\ Centres for Thrombosis and Haemostasis, Institute of Clinical \\ Pathology and Medical Research, NSW Health Pathology, Westmead \\ Hospital, Westmead, New South Wales, Australia \\ ${ }^{3}$ Department of Physiology, Faculty of Medicine, Instituto de \\ Investigación INCLIVA, Fundación Investigación Hospital Clínico, \\ University of Valencia, Valencia, Spain \\ ${ }^{4}$ Leon H. Charney Division of Cardiology, NYU School of Medicine, \\ New York, New York \\ Address for correspondence Giuseppe Lippi, MD, Section of Clinical \\ Biochemistry, University Hospital of Verona, Piazzale LA Scuro 10, \\ 37134 Verona, Italy (e-mail: giuseppe.lippi@univr.it).
}

Semin Thromb Hemost 2018;44:780-786.

\begin{abstract}
Although few doubts remain that physical exercise should be widely promoted for maintenance of health and fitness, the risk of adverse events such as sudden death (especially due to cardiac causes, i.e., sudden cardiac death [SCD]) during exercise remains tangible. The overall risk of sudden death in athletes is relatively low (i.e., usually comprised between 0.1 and $38 / 100,000$ person-years), and globally comparable to that of the general population. However, up to $20 \%$ of all sudden death cases are still recorded while exercising. The most frequent underlying disorders encountered in SCD are hypertrophic cardiomyopathy and coronary artery disease (CAD), representing three quarters of all conditions. The risk related to CAD increases with aging ( $>35$ years old), while that attributable to cardiomyopathies or fatal arrhythmias is especially frequent among young people ( $<35$ years old). Taken together, these findings would lead to the conclusion that physical exercise may be seen as an acute trigger of myocardial ischemia or arrhythmias in some predisposed individuals. Nonetheless, the prevalence of coronary atherosclerosis seems to be higher in athletes than in sedentary subjects with comparable risk profile. On the contrary, coronary plaques in physically active subjects appear more stable, thereby attenuating the risk of rupture and

Keywords

- sudden death

- cardiac arrest

- sports

- physical exercise

- physical activity subsequent myocardial ischemia. These findings, along with evidence of a considerable increase of peak coronary blood flow during exercise, make it very likely that an imbalance between oxygen demand and supply may be the most frequent cause of myocardial ischemia in athletes suffering SCD and/or cardiac arrest. Therefore, all subjects who wish to practice moderate- to high-intensity exercise are recommended to undergo preparticipation screening and annual follow-up.
\end{abstract}

\footnotetext{
* These authors have equal senior authorship on this work.
}

published online June 4, 2018
Issue Theme Hemostasis in Exercise and the Athlete; Guest Editors: Murray J. Adams, BSc(Hons), PhD, MAIMS, FFSc(RCPA) and James W. Fell, BEd, MPhil, PhD.

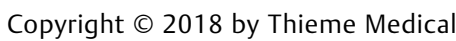
Publishers, Inc., 333 Seventh Avenue, New York, NY 10001, USA. Tel: +1(212) 584-4662.
DOI https://doi.org/ 10.1055/s-0038-1661334. ISSN 0094-6176. 
According to the World Health Organization (WHO), physical activity is currently defined as a bodily movement generated by skeletal muscles and needing energy expenditure, while physical exercise is ranked as a subcategory of physical activity, being planned, repetitive, structured, and focused on obtaining enhancement (or preservation) of physical fitness. ${ }^{1}$ Sports is instead typically defined as an activity based on physical exertion in which individuals or teams compete against others. Sports is additionally divided basically into "amateur" (also known as "recreational"), in which participants are mostly or entirely engaged without remuneration, and "professional" in which athletes are typically remunerated for the time spent in training and/or competing. ${ }^{2}$ Unlike the widespread popular perception, these two definitions do not overlap with those of "competitive" or "noncompetitive" physical exercise, because the aim of athletes engaged in competitive sports involves-or is determined by-rivalry (therefore, entailing the straightforward dichotomy between "winning" and "losing"), whereas subjects engaged in noncompetitive (leisure) exercise are essentially undertaking physical activity for the pleasure of it. The concept of competitive sports is also frequently associated with that of "prize winning," regardless of the real value of the "prize" (i.e., from a simple medal to large amounts of money).

The clear-cut distinction between competitive and noncompetitive sports, as well as that between recreational and professional sports, has relevant implications for human health. Competitive and professional athletes often seek to enhance their athletic performance by high volumes of training, use of dietary supplements, and sometimes unfair practices (e.g., doping), thus pushing their bodies close to, and occasionally over, their physiological limits. ${ }^{3}$ On the contrary, the physiological boundaries of physical exercise, typically expressed in "volume" as the sum of intensity and duration, are much lower for recreational or noncompetitive individuals. However, recreational or noncompetitive individuals may still be exposed to considerable health risks as their volume of physical exercise increases, because their basic training is typically not adequate to sustain acute escalations of exercise volume. ${ }^{4,5}$ The paradigmatic example is that of a recreational runner, who used to run 5 to $10 \mathrm{~km}$ per week, but then decides to participate in a local marathon, covering a distance that is by far in excess of the baseline training status. ${ }^{6}$ Therefore, an appropriate balance between basic training and exercise volume is what mostly defines the safe (or healthy) boundaries of physical activity. This evidence has contributed to developing the concept of "tailored exercise," which entails individually customized activities and volumes of exercise. ${ }^{6,7}$

Recent statistics from the European Commission ${ }^{8}$ identify that approximately $41 \%$ of European citizens are engaged in some forms of physical exercise or sports at least once a week, $46 \%$ of whom practice vigorous physical activity at least once per week, and $20 \%$ exercising $\geq 5$ times per week. Interestingly, nearly three quarters of those practicing vigorous physical exercise admitted to spending $\geq 60$ minutes per week performing such exercise. Overall, the prevalence of physically active men is slightly higher than that of physically active women ( $45 \mathrm{vs.} 37 \%$ ), while the practice of regular exercise declines in parallel with aging (i.e., $64 \%$ in European citizens aged 15-24 years compared with 30\% in those aged 55 years or older). Those with higher levels of education and belonging to higher socioprofessional categories are more likely to exercise. This physical exercise mainly involves parks and other outdoors settings (i.e., $40 \%$ ), followed by in-house (i.e., 36\%) or travel to work, school, and shops (i.e., 25\%). The vast majority (i.e., 74\%) of those who regularly practice physical exercise do not belong to clubs or teams, while the vast majority also engage in physical activity or sports with the aim of improving health and fitness. Interestingly, swimming is the most practiced form of physical activity in Europe (22\%), followed by cycling (19\%), walking (14\%), running (13\%), and football (6\%).

\section{Epidemiology and Causes of Sudden Death in Athletes}

Sudden death in athletes is conventionally defined as an unexpected and instantaneous death occurring during or immediately after (i.e., within 1-3 hours) exercise, due to any cause except violence. ${ }^{9,10}$ Although no definitive classification criteria have been defined so far, it is conventionally accepted that the possible causes of sudden death in sports can be divided into cardiac, that is, sudden cardiac death (SCD), and noncardiac (see - Table 1). Sudden cardiac arrest (SCA) is instead defined as abrupt loss of heart function in subjects with or without heart disease, mainly due to a sudden and unexpected cessation in heartbeat, potentially leading to SCD. ${ }^{11}$

Table 1 Leading causes of sudden death in athletes

\begin{tabular}{|l|}
\hline Noncardiac \\
\hline - Hyperthermia, including heat stroke \\
\hline - Use of illicit drugs \\
\hline $\begin{array}{l}\text { - Acute pulmonary diseases, including pulmonary } \\
\text { embolism }\end{array}$ \\
\hline $\begin{array}{l}\text { - Acute cerebral diseases, including stroke and } \\
\text { hyponatremic encephalopathy }\end{array}$ \\
\hline - Sickle cell disease \\
\hline - Rhabdomyolysis \\
\hline Cardiac \\
\hline - Hypertrophic or dilated cardiomyopathies \\
\hline - Coronary artery abnormalities \\
\hline - Myocarditis \\
\hline - Arrhythmogenic cardiomyopathies, including \\
channelopathies \\
\hline - Valvulopathies \\
\hline - Coronary artery disease \\
\hline - Aortic stenosis or rupture \\
\hline
\end{tabular}


Overall, the percentage of sudden deaths occurring during, or immediately after, exercising is $\sim 5 \%$ of all sudden deaths. ${ }^{12}$ The frequency of sudden death in athletes seems to vary widely in the current scientific literature, depending on sample size, targeted population, geographical area, and definition of sudden death. ${ }^{13}$ Even in endurance sports, such as marathon running, the frequency is highly heterogeneous among published studies. ${ }^{14}$ The number of studies which have so far investigated sudden death in athletes is enormous. A PubMed search using the keywords "sudden death" AND "sport" OR "athletes" with no date or language restrictions produces more than 1,300 documents. Since it is largely infeasible to thoroughly evaluate each single study from such a long list, and given the known heterogeneity of available studies, we will instead report here the findings of some of the most interesting or informative studies based on our knowledge and viewpoint.

In 1980, Maron et al investigated the possible causes of sudden death in 29 competitive young athletes (i.e., aged 13 30 years) by necroscopy, ${ }^{15}$ and found that the vast majority of deaths $(28 / 29 ; 97 \%$ ) were caused by probable ( 6 out of 29 ; $21 \%$ ) or unequivocal (22 out of $29 ; 76 \%$ ) cardiovascular causes. Hypertrophic cardiomyopathy (HCM) was found to be by far the most common abnormality (14 out of $29 ; 48 \%$ ), followed by idiopathic left ventricular hypertrophy ( 5 out of 29; $17 \%$ ) and coronary artery disease (CAD) (3 out of 29; $10 \%)$. In an ensuing investigation published nearly 20 years after this earlier report, Maron et al reported the findings of a large U.S. registry including 1,866 athletes who suddenly died or survived of a SCA while practicing sports over a 27 -year period. ${ }^{16}$ The overall incidence of sudden death was estimated at 0.61/100,000 person-years. Cardiovascular causes could be identified in more than $56 \%$ of cases $(1,049$ out of 1,866$)$, the most frequent of which were possible or certain HCM (370 out of 1,866; $19.8 \%$ ), myocarditis (57 out of 1,$866 ; 3.1 \%$ ), and arrhythmogenic right ventricular cardiomyopathy (ARVC) (41 out of 1,$866 ; 2.2 \%$ ). CAD was identified in 23 out of $1,866(1.2 \%)$ cases, while the most frequent noncardiac causes (excluding injuries) were heat stroke, abuse of illicit drugs, and acute pulmonary diseases. Most cases were recorded in American football athletes, followed by basketball, soccer, and baseball players. A third study was then published by the same team of authors in 2016, based on data of 2,046 athletes contained in the U.S. National Registry of Sudden Death in Athletes accumulated between the years 1980 and $2011 .{ }^{17}$ Autopsyconfirmed cardiovascular diseases could be identified in 802 out 2,046 (40\%) cases, mainly represented by HCM (302 out of 2,$046 ; 14.8 \%$ ), coronary arteries abnormalities (158 out 2,$046 ; 7.7 \%$ ), myocarditis (57 out of 2,$046 ; 2.8 \%$ ), ARVC (43 out of 2,$046 ; 2.1 \%$ ), and CAD (38 out of 2,$046 ; 1.9 \%$ ). The most frequently involved sports disciplines were football, basketball, baseball, and cross-country running. Interestingly, the most frequent noncardiovascular- and noninjury-related causes of sudden death were illicit drug abuse ( 81 out of 2,$046 ; 4.0 \%$ ) and sickle cell disease (31 out of 2,$046 ; 1.5 \%$ ). The vast majority of subjects with confirmed SCD were noncompetitive athletes (746 out of $842 ; 88.6 \%$ ).
A remarkably high frequency of cardiovascular disorders ( 163 out of $200 ; 81.5 \%$ ), followed by cerebral ( 15 out of 200 ; $7.5 \%$ ) and pulmonary (10 out of $200 ; 5 \%$ ) diseases was found in 200 cases of sudden death in young Italian athletes aged 35 years or younger. ${ }^{18}$ The most common cardiovascular causes of SCD were CAD (23\%), followed by arrhythmogenic cardiomyopathies (22\%), valvulopathies $(10 \%)$, and $\mathrm{HCM}$ (5.5\%).

Recently, Harmon et al published the results of a large survey based on the U.S. National Collegiate Athletic Association database, in which all cases of sudden death involving collegial athletes were identified during a 5-year period (years 2004-2008). ${ }^{19}$ Overall, 273 deaths could be recorded during 1,969,663 athlete participant-years (i.e., 13.9/100,000 person-years), with 80 medical deaths (i.e., excluding injuries and suicides; 4.1/100,000 person-years). Cardiac causes were identified in more than half of the cases ( 45 out of 80; $56 \%$ ), while other important causes were heat stroke and sickle cell disease. The most involved sports was basketball, followed by football and swimming.

In a large epidemiological study aimed to define incidence and outcome of SCA in athletes engaged in marathon and half-marathon running in the United States over a 10 -year period, the overall frequency of SCA was $0.54 / 100,000$ person-years, nearly fourfold higher in athletes engaged in marathons than in half-marathon running (i.e., $1.01 \mathrm{vs.}$ $0.27 / 100,000$ person-years). ${ }^{20}$ The leading causes of SCA were HCM (49\%) and CAD (16\%), followed by arrhythmias (14\%), hyponatremia and hyperthermia (both $7 \%$ ), and other cardiomyopathies (3\%). In another large prospective study on middle-aged (i.e., 35-65 years) residents of a large U.S. community, an overall of 1,247 cases of SCD could be identified, 63 (5\%) of which occurring during sports activities (incidence, 2.2/100,000 person-years). ${ }^{21}$ Interestingly, the leading underlying cause of SCD was CAD (36 out of 63; $57.1 \%$ ), while a positive history of heart disease could also be recorded in 20 out of 63 (31.7\%) cases.

Chappex et al published an interesting retrospective study based on autopsy records and aimed to compare the possible triggers of SCD related and nonrelated to physical exercise. ${ }^{22}$ Overall, exercise-related sudden deaths were substantially lower than those that were nonexercise-related (i.e., 12 vs. $88 \%$ ). In both populations, CAD was the most commonly encountered abnormality. However, in 357 cases of sudden death collected over a 10-year period in United Kingdom athletes, ${ }^{23}$ arrhythmogenic cardiomyopathies were the most frequent causes of SCD (55\%), followed by left ventricular hypertrophy (22\%), coronary arteries abnormalities (5\%), and CAD (2\%). The sports disciplines most involved were running (92 out of $357 ; 25.8 \%$ ) and football (91 out of $357 ; 25.5 \%$ ), followed by cycling and gymnastics (both 30 out of $357 ; 8.4 \%$ ) and swimming (22 out of $357 ; 6.2 \%$ ). The vast majority of subjects were competitive athletes ( 245 out of $357 ; 68.6 \%$ ).

In 2016, Risgaard collected data from death certificates, autopsy reports, discharge summaries, and registries in Denmark, to identify all possible cases of sudden death that occurred between 2007 and 2009. ${ }^{24}$ Overall, 881 cases could be identified, $44(5.0 \%)$ of which were attributable to 
exercise (11 in competitive athletes and 33 in noncompetitive athletes). In subjects aged less than 50 years, the overall frequency of SCD was $8.6 / 100,000$ person-years, increasing to $38.5 / 100,000$ person-years in subjects aged 48 to 49 years. The most common causes of sudden death were CAD (158 out of $881 ; 17.9 \%)$, followed by sudden unexplained mortality $(136$ out of $881 ; 15.4 \%)$. Notably, no significant difference in the frequency of sudden death could be observed between noncompetitive and competitive athletes. Most exerciserelated events occurred in runners, followed by cyclists and soccer players. Similarly, among nine cases of sudden death or SCA registered over a 25-year period (i.e., from 1990 to 2014) in a 14-km running event annually held in Sydney (Australia), ${ }^{25}$ yielding a frequency of $3 / 100,000$ personyears, a heart attack was identified as the cause of SCD in the majority of cases (five out of nine; 56\%), followed by SCA (four out of nine; 34\%). On those who had an autopsy performed, CAD was present in the majority of cases (27 out of $61 ; 44 \%){ }^{25}$ Likewise, among triathlon participants from the U.S. National Registry of Sudden Death in Athletes followed up for 30 years, ${ }^{26} 135$ cases of sudden death or SCA were identified (1.74/100,000 person-years), mostly occurring during the swimming trial (90 out of $135 ; 67 \%$ ), while only 8 cases ( $6 \%$ ) could be recorded during postrace recovery.

In 2017, Landry et al published the results of a large retrospective investigation based on data of the Rescu Epistry cardiac arrest database (subjects aged between 12 and 45 years), and averaging 18.5 million person-years of observation. ${ }^{27}$ Overall, 74 SCA were recorded during sports participation, 58 occurring in competitive sports and 16 in noncompetitive sports, thus leading to a global incidence of 0.76 SCA per 100,000 athlete-years. The leading cause of SCA in athletes aged 35 years or younger was a structural and primary arrhythmia, while CAD was the leading cause in those aged 35 to 45 years. The authors concluded that the risk of SCA was relatively low in athletes participating in sports activities compared with the incidence in the general population of the same age group (i.e., 4.84 cases per 100,000 person-years).

Accordingly, some interesting conclusions can therefore be made based on published information on sudden death or SCA in athletes. First, the overall risk of sudden death in athletes, albeit relatively low (usually comprised between 0.1 and $38 / 100,000$ person-years) and globally comparable to that of the general population, ${ }^{9}$ remains significant since up to $20 \%$ of all sudden deaths occur while exercising. The most frequent underlying disorders encountered in SCD are HCM and $C A D$ representing altogether over three quarters of all conditions. Interestingly, the risk related to CAD increases with aging ( $>35$ years old), while that attributable to cardiomyopathies and/or fatal arrhythmias is especially frequent among younger individuals ( $<35$ years old).

\section{Pathogenesis of Ischemic Myocardial Injury in Physical Exercise}

Many of the previously described studies showed that obstructive CAD is one of the leading underlying conditions encountered in athletes who died or experienced of a SCA while exercising, becoming the main cause of SCD in subjects aged 35 years or older. ${ }^{28,29}$ Obstructive CAD is the result of a progressively worsening coronary atherosclerosis, which leads to a partial or total occlusion of the blood vessel. A superimposed arterial thrombosis and a disruption in oxygen supply to myocardial tissue occur when the degree of vessel stenosis is high (i.e., > 75\%). This phenomenon may be accompanied, or not, by ulceration or complete rupture of atherosclerotic plaque. ${ }^{30,31}$ According to the most recent classification, this is now defined as the "typical" ("type 1 ") myocardial infarction, while the so-called type 2 myocardial infarction recognizes distinctive mechanisms leading to irreversible ischemic myocardial injury, mainly attributable to imbalance between oxygen demand from the contracting myocardium and oxygen supply. ${ }^{32}$ Recent studies have shown that a transitory myocardial injury is almost physiological in endurance athletes and is directly related to exercise volume. ${ }^{33}$ Nevertheless, when oxygen supply is no longer sufficient to withstand the enhanced cardiac demand characterizing physical exercise (coronary blood flow may increase up to fivefold during heavy exercise), ${ }^{34}$ oxygen availability dramatically drops and the ischemic damage becomes gradually irreversible (i.e., myocardial infarction with necrosis of myocardial tissue). This can actually be attributable to at least three biological mechanisms, as shown in - Fig. $\mathbf{1}^{35}$

The first mechanism is a likely consequence of vasoconstriction caused by an intense release of catecholamines, up to the so-called adrenaline rush, occurring during stress and strenuous efforts. ${ }^{36}$ This condition, also known as "Takotsubo syndrome", is likely attributable to catecholamineinduced coronary vasoconstriction and/or spasm combined with enhanced cardiac workload, ultimately leading to ischemic myocardial injury in a setting of a coronary tree with no, or only modest presence of, atherosclerotic lesions on angiography (- Fig. 1). ${ }^{37,38}$

The second mechanism is principally attributable to the presence of modest CAD (i.e., even $<25-50 \%$ ), which would not be sufficient to trigger an irreversible ischemic injury in resting conditions, but which may instead become clinically significant as the oxygen demand by the contracting myocardium substantially increases during heavy physical exercise (-Fig. 1). ${ }^{34}$ This is probably the most typical mechanism underlying SCD cases recorded among "weekend warriors" or in occasional endurance runners. ${ }^{29}$ It is also interesting to note here that physical exercise acutely triggers many prothrombotic changes, including enhanced thrombin generation and platelet hyperreactivity, which may ultimately amplify the severity of coronary thrombosis. $^{39}$

The third mechanism is substantially independent of the structure and function of the coronary tree, but is attributable to insufficient oxygen availability in blood, which becomes much more clinically significant during heavy exercise. The most typical condition is acute or chronic anemia, in which the concentration of hemoglobin transported within the erythrocytes is no longer sufficient to meet 


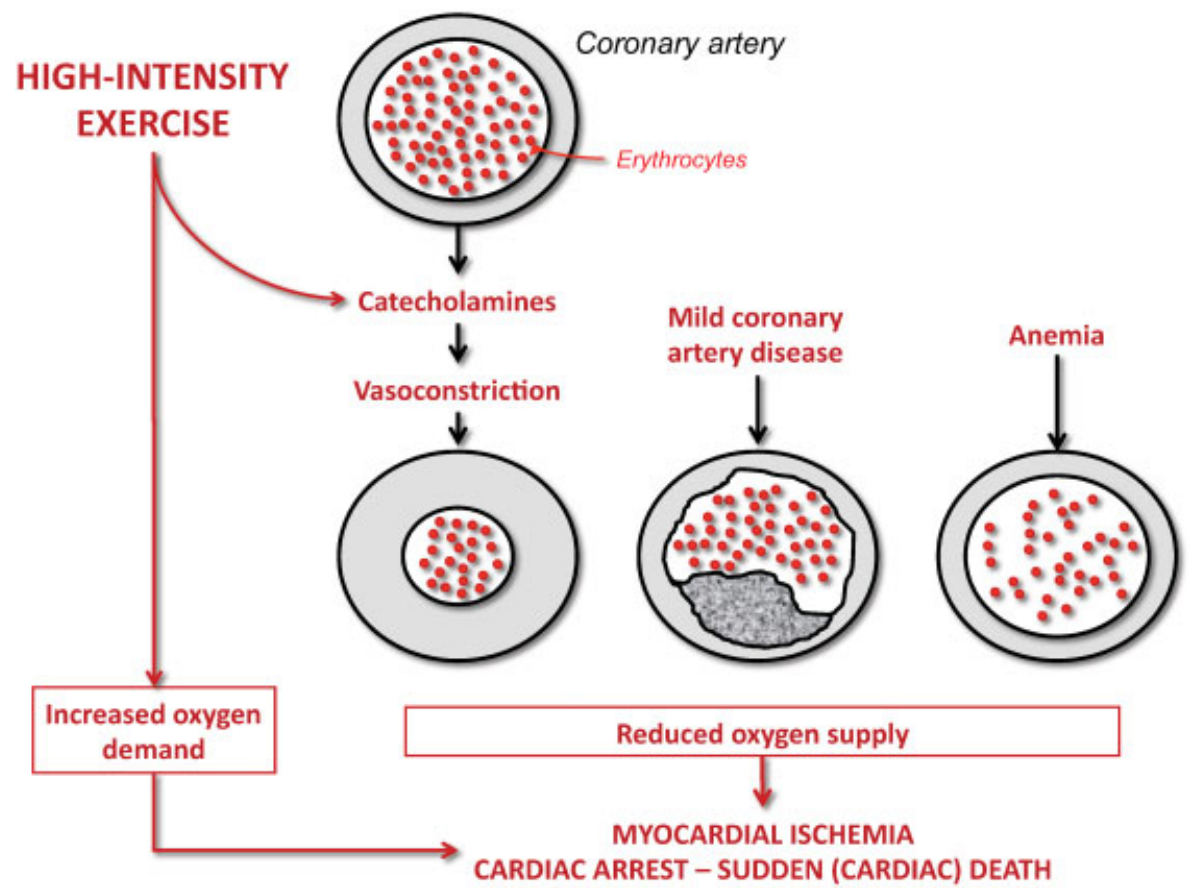

Fig. 1 Possible mechanisms leading to myocardial ischemia during physical exercise.

the enhanced oxygen demand of contracting myocardium, thus leading to irreversible ischemic injury even in the lack of significant CAD or superimposed thrombosis (-Fig. 1). ${ }^{40}$

Notably, this classification of type 2 myocardial infarction is not always straightforward because an overlap among the three mechanisms may occur in certain individuals (i.e., catecholamines-induced vasoconstriction may also occur in patients with modest coronary atherosclerosis), nor can it be excluded that some athletes may actually suffer from a typical type 1 myocardial infarction. Two recent studies published by Merghani et $\mathrm{al}^{41}$ and by Aengevaeren et $\mathrm{al}^{42}$ showed that though the prevalence of coronary atherosclerosis seems to be higher in athletes than in sedentary subjects with comparable risk profile, coronary plaques in physically active people appear more stable in nature, so attenuating the risk of rupture and subsequent myocardial ischemia. These findings, along with evidence of a considerable increase of peak coronary blood flow during exercise, make it very likely that type 2 myocardial infarction may be the most frequent cause of myocardial ischemia in athletes with SCA and/or SCD (- Fig. 1).

\section{Preventing Sudden Cardiac Death during and After Physical Exercise}

According to the $\mathrm{WHO},{ }^{43}$ physical exercise remains one of the leading measures for preventing the onset and the complications of a large number of the most frequent human disorders (i.e., coronary heart disease, stroke, diabetes, cancer, depression, fractures), but is also essential for weight maintenance, increasing fitness, and for decreasing the risk of allcause mortality. The WHO currently recommends that adults (i.e., between 18 and 64 years) should be engaged in not less than 150 minutes of moderate-intensity aerobic physical exercise or in not less than 75 minutes of vigorous-intensity aerobic physical activity per week, performed in bouts of not less than 10 minutes duration. Additional health benefits may be achieved with engagement in moderate-intensity aerobic physical activity up to 300 minutes or in 150 minutes of vigorous-intensity aerobic physical activity throughout the week. Nearly identical recommendations have been provided by the American Heart Association. ${ }^{44}$

Although little doubts exist that these straightforward recommendations should be widely promoted for maintenance of health and fitness, the risk of adverse events (especially SCD and/or cardiac arrest) during exercise remains tangible, as highlighted by the current scientific literature. Therefore, the gap between the putative benefits and the possible risks of physical exercise should be closed by developing additional recommendations, especially aimed to identify subjects at enhanced risk of adverse events while exercising. ${ }^{45}$ Interestingly, more than $50 \%$ of middle-aged individuals who suffer SCA have warning symptoms up to 4 weeks before the fatal event. ${ }^{46}$ In this regard, preparticipation screening (PPS), an approach aimed to identify athletes at risk of SCA and/or SCD, is highly recommended by the American Heart Association, the European Society of Cardiology, and the International Olympic Committee ${ }^{6}$ because it is the most effective strategy to prevent these events. ${ }^{47}$ Likewise, education during screening procedure by health professionals also seems recommended, instructing subjects to reach realistic goals in terms of performance and exercise safely by planning the optimal individually tailored training program, as well as to be aware of warning signs or symptoms of cardiovascular disease (angina pectoris, ischemic equivalents, palpitations, impaired consciousness), or sudden appearance of a 
cardiovascular event associated with exercise. Therefore, from our point of view, implementing and/or reinforcing the recommendation of undergoing PPS, both in competitive and noncompetitive athletes, and providing appropriate education during or immediately after screening, may reflect a good practice standard to adopt. Briefly, PPS in young population includes family history, physical examination, and a 12-lead electrocardiogram. ${ }^{47}$ In adults, however, the risk calculation using scoring methods (based on age, sex, blood pressure, blood cholesterol, and smoking history) is also recommended. ${ }^{47,48}$ Last but not least, undergoing an annual follow-up with maximal exercise testing (and even other cardiologic evaluations such as an echocardiogram) is also advised in elderly and middle-aged individuals with an increased risk for coronary events who wish to practice moderate/intense exercise. ${ }^{6,47,49}$ Finally, no definitive evidence currently exists about the clinical efficiency of performing additional investigations such as blood and genetic testing, with the exception of urinalysis and iron stores assessment in female athletes. ${ }^{49}$

\section{Conclusion}

Although there is no doubt that exercise produces a wide range of benefits for maintenance of health and fitness, some individuals may still be at risk of adverse events, including SCA and/or SCD. It has been extensively demonstrated that PPS performed by trained clinicians represents an efficient strategy for prevention of SCA and/or SCD, allowing the identification of asymptomatic subjects with potentially lethal cardiovascular abnormalities and reducing the death rate. In conclusion, all subjects who wish to practice moderate- to high-intensity exercise are highly recommended to undergo PPS and annual follow-up.

\section{Conflict of Interest}

None.

\section{References}

1 World Health Organization. Physical activity. Available at: http:// www.who.int/topics/physical_activity/en/. Accessed February 2, 2018

2 Allison L. Amateurism in Sport: An Analysis and a Defence. London, UK: Frank Cass \& Co; 2001

3 Lippi G, Franchini M, Guidi GC. Doping in competition or doping in sport? Br Med Bull 2008;86:95-107

4 Warburton DE, Nicol CW, Bredin SS. Health benefits of physical activity: the evidence. CMAJ 2006;174(06):801-809

5 Lippi G, Schena F, Guidi GC. Health benefits of physical activity. CMAJ 2006;175(07):776, author reply 777

6 Sanchis-Gomar F, Santos-Lozano A, Garatachea N, et al. My patient wants to perform strenuous endurance exercise. What's the right advice? Int J Cardiol 2015;197:248-253

7 Müller-Riemenschneider F, Reinhold T, Nocon M, Willich SN. Long-term effectiveness of interventions promoting physical activity: a systematic review. Prev Med 2008;47(04):354-368

8 European Commission. Special Eurobarometer 412 "Sport and Physical Activity". 2014. DOI: 10.2766/73002

9 Montagnana M, Lippi G, Franchini M, Banfi G, Guidi GC. Sudden cardiac death in young athletes. Intern Med 2008;47(15):1373-1378
10 Montagnana M, Lippi G, Franchini M, Targher G, Cesare Guidi G. Sudden cardiac death: prevalence, pathogenesis, and prevention. Ann Med 2008;40(05):360-375

11 American Heart Association. Heart Attack or Sudden Cardiac Arrest: How Are They Different? Available at: http://www. heart.org/HEARTORG/Conditions/More/CardiacArrest/AboutCardiac-Arrest_UCM_307905_Article.jsp\#.WmtGTuHhDcs. Accessed February 2, 2018

12 Narayanan K, Bougouin W, Sharifzadehgan A, et al. Sudden cardiac death during sports activities in the general population. Card Electrophysiol Clin 2017;9(04):559-567

13 Asif IM, Harmon KG. Incidence and etiology of sudden cardiac death: new updates for athletic departments. Sports Health 2017; 9(03):268-279

14 Waite O, Smith A, Madge L, Spring H, Noret N. Sudden cardiac death in marathons: a systematic review. Phys Sportsmed 2016; 44(01):79-84

15 Maron BJ, Roberts WC, McAllister HA, Rosing DR, Epstein SE. Sudden death in young athletes. Circulation 1980;62(02):218-229

16 Maron BJ, Doerer JJ, Haas TS, Tierney DM, Mueller FO. Sudden deaths in young competitive athletes: analysis of 1866 deaths in the United States, 1980-2006. Circulation 2009;119(08):1085-1092

17 Maron BJ, Haas TS, Ahluwalia A, Murphy CJ, Garberich RF. Demographics and epidemiology of sudden deaths in young competitive athletes: from the United States National Registry. Am J Med 2016;129(11):1170-1177

18 Basso C, Corrado D, Thiene G. Cardiovascular causes of sudden death in young individuals including athletes. Cardiol Rev 1999;7 (03):127-135

19 Harmon KG, Asif IM, Klossner D, Drezner JA. Incidence of sudden cardiac death in National Collegiate Athletic Association athletes. Circulation 2011;123(15):1594-1600

20 Kim JH, Malhotra R, Chiampas G, et al; Race Associated Cardiac Arrest Event Registry (RACER) Study Group. Cardiac arrest during long-distance running races. N Engl J Med 2012;366(02):130-140

21 Marijon E, Uy-Evanado A, Reinier K, et al. Sudden cardiac arrest during sports activity in middle age. Circulation 2015;131(16): 1384-1391

22 Chappex N, Schlaepfer J, Fellmann F, Bhuiyan ZA, Wilhelm M, Michaud K. Sudden cardiac death among general population and sport related population in forensic experience. J Forensic Leg Med 2015;35:62-68

23 Finocchiaro G, Papadakis M, Robertus JL, et al. Etiology of sudden death in sports: insights from a United Kingdom Regional Registry. J Am Coll Cardiol 2016;67(18):2108-2115

24 Risgaard B. Sudden cardiac death: a nationwide cohort study among the young. Dan Med J 2016;63(12):B5321

25 Sweeting J, Ingles J, Ball K, Semsarian C. Sudden deaths during the largest community running event in Australia: a 25-year review. Int J Cardiol 2016;203:1029-1031

26 Harris KM, Creswell LL, Haas TS, et al. Death and Cardiac Arrest in U.S. Triathlon Participants, 1985 to 2016: a case series. Ann Intern Med 2017;167(08):529-535

27 Landry CH, Allan KS, Connelly KA, Cunningham K, Morrison LJ, Dorian P; Rescu Investigators. Sudden cardiac arrest during participation in competitive sports. N Engl J Med 2017;377(20):1943-1953

28 Coplan NL, Gleim GW, Nicholas JA. Exercise and sudden cardiac death. Am Heart J 1988;115(1 Pt 1):207-212

29 Sheppard MN. Aetiology of sudden cardiac death in sport: a histopathologist's perspective. Br J Sports Med 2012;46(Suppl 1): i15-i21

30 Lippi G, Franchini M, Targher G. Arterial thrombus formation in cardiovascular disease. Nat Rev Cardiol 2011;8(09):502-512

31 Lippi G, Favaloro EJ. Venous and arterial thromboses: two sides of the same coin? Semin Thromb Hemost 2018;44(03):239-248

32 Cervellin G, Mattiuzzi C, Bovo C, Lippi G. Diagnostic algorithms for acute coronary syndrome-is one better than another? Ann Transl Med 2016;4(10):193 
33 Lippi G, Cervellin G, Schena F. How much myocardium mass may be injured during endurance physical exercise? Clin Chim Acta 2017;470:29-30

34 Duncker DJ, Bache RJ. Regulation of coronary blood flow during exercise. Physiol Rev 2008;88(03):1009-1086

35 Lippi G, Sanchis-Gomar F, Cervellin G. Chest pain, dyspnea and other symptoms in patients with type 1 and 2 myocardial infarction. A literature review. Int J Cardiol 2016;215:20-22

36 Schömig A. Catecholamines in myocardial ischemia. Systemic and cardiac release. Circulation 1990;82(3, Suppl):II13-II22

37 Pelliccia F, Kaski JC, Crea F, Camici PG. Pathophysiology of Takotsubo syndrome. Circulation 2017;135(24):2426-2441

38 Hung MJ, Hung MY, Cheng CW, Yang NI, Cherng WJ. Clinical characteristics of patients with exercise-induced ST-segment elevation without prior myocardial infarction. Circ J 2006 70(03):254-261

39 Lippi G, Maffulli N. Biological influence of physical exercise on hemostasis. Semin Thromb Hemost 2009;35(03):269-276

40 Bailey D, Aude YW, Gordon P, Burtt D. ST-segment elevation myocardial infarction, severe anemia and nonobstructive coronary disease: case report and brief comment. Conn Med 2003 67(01):3-5

41 Merghani A, Maestrini V, Rosmini S, et al. Prevalence of subclinical coronary artery disease in masters endurance athletes with a low atherosclerotic risk profile. Circulation 2017;136(02): 126-137
42 Aengevaeren VL, Mosterd A, Braber TL, et al. Relationship between lifelong exercise volume and coronary atherosclerosis in athletes. Circulation 2017;136(02):138-148

43 World Health Organization. Physical Activity and Adults. Available at: http://www.who.int/dietphysicalactivity/factsheet_ adults/en/. Accessed February 2, 2018

44 American Heart Association. Recommendations for Physical Activity in Adults. Available at: http://www.heart.org/HEARTORG/HealthyLiving/PhysicalActivity/FitnessBasics/American-Heart-AssociationRecommendations-for-Physical-Activity-in-Adults_UCM_ 307976_Article.jsp\#.WmytbXxG0dU. Accessed February 2, 2018

45 Emery MS, Kovacs RJ. Sudden cardiac death in athletes. JACC Heart Fail 2018;6(01):30-40

46 Marijon E, Uy-Evanado A, Dumas F, et al. Warning symptoms are associated with survival from sudden cardiac arrest. Ann Intern Med 2016;164(01):23-29

47 Corrado D, Zorzi A. Sudden death in athletes. Int J Cardiol 2017; 237:67-70

48 Borjesson M, Urhausen A, Kouidi E, et al. Cardiovascular evaluation of middle-aged/ senior individuals engaged in leisure-time sport activities: position stand from the sections of exercise physiology and sports cardiology of the European Association of Cardiovascular Prevention and Rehabilitation. Eur J Cardiovasc Prev Rehabil 2011;18(03):446-458

49 Chugh SS, Weiss JB. Sudden cardiac death in the older athlete. J Am Coll Cardiol 2015;65(05):493-502 